\title{
DESIGN OF ALLOY'S CONCENTRATIONS FOR OPTIMIZED STRENGTH, TEMPERATURE, TIME-TO-RUPTURE, COST AND WEIGHT
}

\author{
George S. Dulikravich ${ }^{1}$, Igor N. Egorov-Yegorov², \\ ${ }^{1}$ Department of Mechanical and Materials Engineering, EC 3474 \\ MAIDROC Laboratory, Florida International University, College of Engineering, \\ 10555 West Flagler Street, Miami, Florida 33174, U. S. A. \\ ${ }^{2}$ IOSO Technology Center; Milashenkova Ulitsa 10-201, Moscow 127322, RUSSIA
}

Keywords: superalloys, multi-objective optimization, alloys design

\begin{abstract}
A novel method has been developed and experimentally verified that can enable a significant part of the steel alloy development procedure to be performed computationally by using the power of a true mathematical evolutionary multi-objective optimization algorithm. During the alloy optimization process, maximized operating temperature, tensile stress, time-to-rupture, and minimized cost and weight were treated as simultaneous often conflicting objectives. Concentrations of most important alloying elements were predicted so that new alloys have the best multiple properties. This alloy design concept was verified using strictly experimental data. The number of required experimental evaluations of the candidate alloys with this optimization approach is very low. This approach has the potential of identifying the new chemical compositions of significantly superior steel alloys with only a few hundred alloy samples.
\end{abstract}

\section{Introduction}

A brute-force optimization of thermo-mechanical properties of a steel alloy by varying its chemical composition with $\mathrm{N}$ alloying elements would involve creating an $\mathrm{N}$-dimensional matrix of alloy compositions and then interpolating and searching for the extreme points in such a matrix. If concentration of each alloying element is to be varied within a specified range, this variation could be approximated by, say, ten parameters. This means that in the case of an alloy with six alloying elements, this "optimization" would require determining properties of $10 * * 6=$ $1,000,000$ different alloy samples. Typically, this means that each of these alloys would have to be cast and tested on a laboratory scale which is obviously unfeasible.

In order to reduce the time and cost of the acquisition of such a data bank, there has been a strong effort to develop and use several very complex mathematical models that are based on non-equilibrium thermodynamics of solids, thus minimizing the need for manufacturing and experimental evaluation of the actual alloy samples. However, the exclusive use of this strictly computational approach based on artificial neural networks (ANNs) [1] and more recently on genetic algorithms [2] has been shown to possess dubious reliability and versatility as recently frankly demonstrated by Bhadeshia and Sourmail [3]. ANNs are efficient and relatively accurate interpolating algorithms for any multi-parameter function $[1,3]$. But, ANNs are not efficient and 
accurate search algorithms and they are definitely not extrapolation algorithms. That is, the use of ANNs alone is not reliable for "getting out of the box" search outside the given data set. Therefore, it is important to understand a need for a mathematically sound multi-objective stochastic optimization algorithm capable of finding the global minimum and confidently search outside a given initial data base.

\section{Multi-Objective Optimization Algorithm - IOSO}

The key to the success of our alloy design methodology is the robustness, accuracy, and efficiency of the multi-objective constrained optimization algorithm so that it does not require an extremely large number of objective function (mechanical and physical properties of alloys) evaluations, which makes the total number of experimental evaluations unacceptably large.

The multi-objective optimization problem maximizes a vector of objective functions subject to a vector of inequality constraints and a vector of equality constraints. The solution of this problem is not unique. With the introduction of the Pareto dominance concept [4] the solutions belonging to the non-dominated group are the ones for which it is not possible to improve any individual objective without deteriorating the values of at least some of the remaining objectives. Gradientbased optimization algorithms are not suitable for Pareto optimization. Because of the unknown topology of the multi-objective function space possibly having multiple extrema, the multiobjective optimization is typically performed with evolutionary non-gradient based algorithms.

Our approach is based on the use and a special adaptation of a stochastic, multi-objective constrained Indirect Optimization based upon Self-Organization (IOSO) algorithm [5, 6]. IOSO stochastic multi-objective optimization algorithm allows for concentrations of a number of alloying elements to be optimized so that a finite number of properties (maximum tensile strength, maximum operating temperature, maximum time-until-rupture, minimum weight, minimum cost, etc.) of the alloy are simultaneously extremized [7-11]. The main benefits of this optimizer are its outstanding reliability in avoiding local minimums, its computational speed, and a remarkably small number of required experimental evaluations of alloy samples as compared to more traditional semi-stochastic optimizers such as genetic algorithms. IOSO uses a multidimensional response surface technique with adaptive global and middle-range multi-point approximation. The self-adapting response surface formulation [6] used by IOSO allows for incorporation of realistic non-smooth variations of experimentally obtained data and allows for accurate interpolation of such data $[5,6]$. The first stage of IOSO is the creation of approximations of the objective functions. Each iteration in this stage represents a decomposition of the initial approximation function into a set of simple approximation functions so that the final response function is a multi-level graph. The second stage of IOSO algorithm is the optimization of these approximation functions. This approach allows for corrective updates of the structure and the parameters of the response surface approximation. During each iteration of IOSO, the optimization of the response function is performed only within the current search area. This step is followed by manufacturing and experimentally evaluating the thermo-mechanical properties for each of the predicted optimized alloy compositions. During the IOSO operation, the information concerning the behavior of the objective function in the vicinity of the extremum is stored, and the response function is made more accurate for this specific search area only.

One of the advantages of the proposed alloy optimization approach is the possibility of ensuring good approximating capabilities using a minimum amount of available information. In a recent example demonstrating the potential of this alloy design methodology [9-11], an initial data base, containing 120 steel alloys was generated using Sobol's algorithm [12]. The chemical compositions of these alloys were determined so that they are as uniformly distributed in the function space as possible, thus, creating conditions for very accurate response surface fit. 

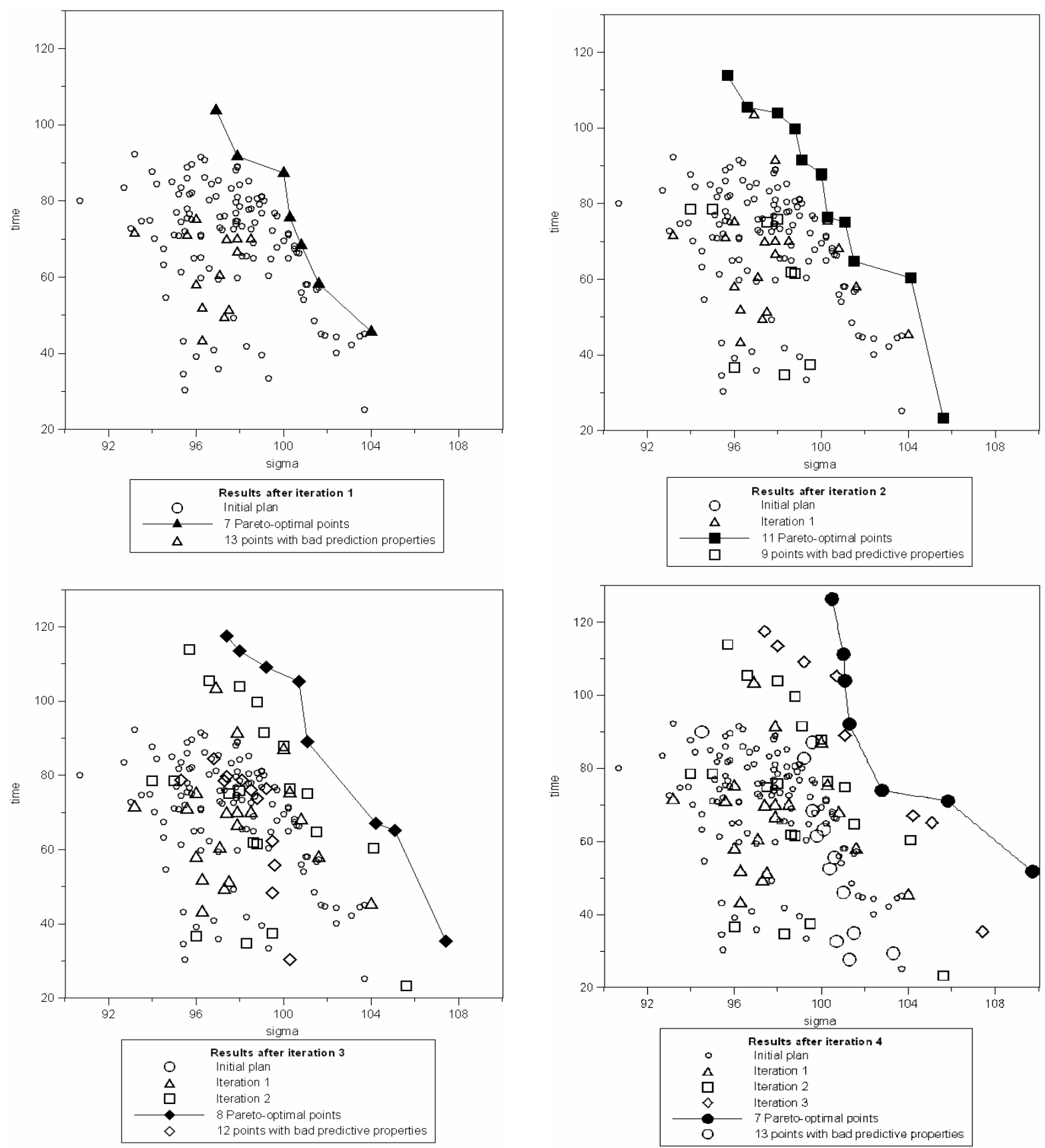

Figure 1: Experimental data for time-to-rupture at 975 degrees Celsius and stress of $23 \mathrm{~kg} / \mathrm{mm}^{2}$ vs. maximum tensile stress at room temperature for the 120 original Ni-base alloys (circles) and the 20 Pareto optimized new alloys (other symbols) after each of the four iterations using IOSO optimization algorithm. The experimentally confirmed Pareto optimal alloys after each optimization cycle are depicted with dark symbols.

Table I. Specified ranges of design variables (concentrations) of 7 major alloying elements

\begin{tabular}{|c|c|c|c|c|c|c|c|}
\hline & $\mathbf{C}$ & $\mathbf{C r}$ & $\mathbf{C o}$ & $\mathbf{W}$ & $\mathbf{M o}$ & $\mathbf{A l}$ & $\mathbf{T i}$ \\
\hline $\min$ & 0.13 & 8.0 & 9.0 & 9.5 & 1.2 & 5.1 & 2.0 \\
\hline $\max$ & 0.20 & 9.5 & 10.5 & 11.0 & 2.4 & 6.0 & 2.9 \\
\hline
\end{tabular}

These 120 Ni-base steel alloys were then cast, each having its own specific concentration of alloying elements. Heat treatment of the samples of such alloys involved heating to $1210 \mathrm{C}$, holding for 4 hours, and air cooling to room temperature. The chemical elements deemed to be important were $\mathrm{Ni}, \boldsymbol{C}, \boldsymbol{C r}, \boldsymbol{C o}, \boldsymbol{W}, \mathbf{M o}, \boldsymbol{A l}, \boldsymbol{T i}, \boldsymbol{B}, \boldsymbol{N b}, \boldsymbol{C e}, \boldsymbol{Z r}, \boldsymbol{Y}$, while elements such as S, P, Fe, 
$\mathrm{Mn}, \mathrm{Si}, \mathrm{Pb}, \mathrm{Bi}$ were treated as extraneous impurities. Chemical elements whose concentrations were optimized were Ni, C, Cr, Co, W, Mo, Al, Ti (Table I). Concentrations of Nb, B, Ce, Zr, Y in all sample alloys were kept constant at $1.1 \%, 0.025 \%, 0.015 \%, 0.04 \%$, and $0.01 \%$, respectively [10]. Two simultaneous objectives of the alloy concentration optimization process were: maximize stress at room temperature, and maximize time until rupture at 975 degrees Celsius with a fixed stress of $23 \mathrm{~kg} / \mathrm{mm}^{2}$. The experimental evaluation of the stress and life until rupture at a fixed temperature were performed for each of these 120 alloys (Fig. 1). Using this experimental data set, a multi-objective optimization problem was solved with IOSO software that found 20 Pareto optimized alloys which were then manufactured and experimentally tested. Seven of these 20 new alloys obtained after the first iteration with IOSO were found to belong to the Pareto optimal set. That is, we experimentally confirmed that 7 out of 20 predicted optimized steel alloy compositions can sustain higher stress and last longer at elevated temperature than any of the original 120 alloys (Fig. 1). The remaining 13 predicted optimized alloys were experimentally found not to belong to a Pareto set, but fell into the general quality of the original 120 alloys. Then, we repeated the multi-objective optimization problem using 120 original plus 20 newly predicted alloys. IOSO optimizer again predicted concentrations and properties of 20 new alloys. They were then manufactured and tested using classical experimental techniques confirming that 11 of these 20 alloys are indeed superior to any of the original 120 alloys and any of the first 20 predicted alloys. This constituted the second iteration. The third and fourth iterations were repetitions of this cycle. Figure 1 demonstrates that this multi-objective optimization combined with exclusively experimental verification creates significantly better new alloys and requires an acceptably low number of these alloys to be manufactured and tested.

\section{Incorporating Cost and Weight of an Alloy}

We obtained a standard daily price list of typical alloying elements available on the metals market and a list of densities of these alloying elements. The original idea was to optimize simultaneously the following five objectives: maximum stress, maximum temperature, time-torupture, minimum cost of the raw ingredients, and minimum volume-specific weight (density) of the resulting steel alloy. However, we reformulated this as a sequence of different two-objective optimization problems where remaining objectives are treated as constraints (Figs. 2-5). Each symbol in these figures represents an optimized alloy with its own chemical composition. Notice that by a similar use of the constraints this approach has the potential for designing alloys with specified multiple properties, thereby maximizing their utilization at reduced cost $[8,10,11]$.

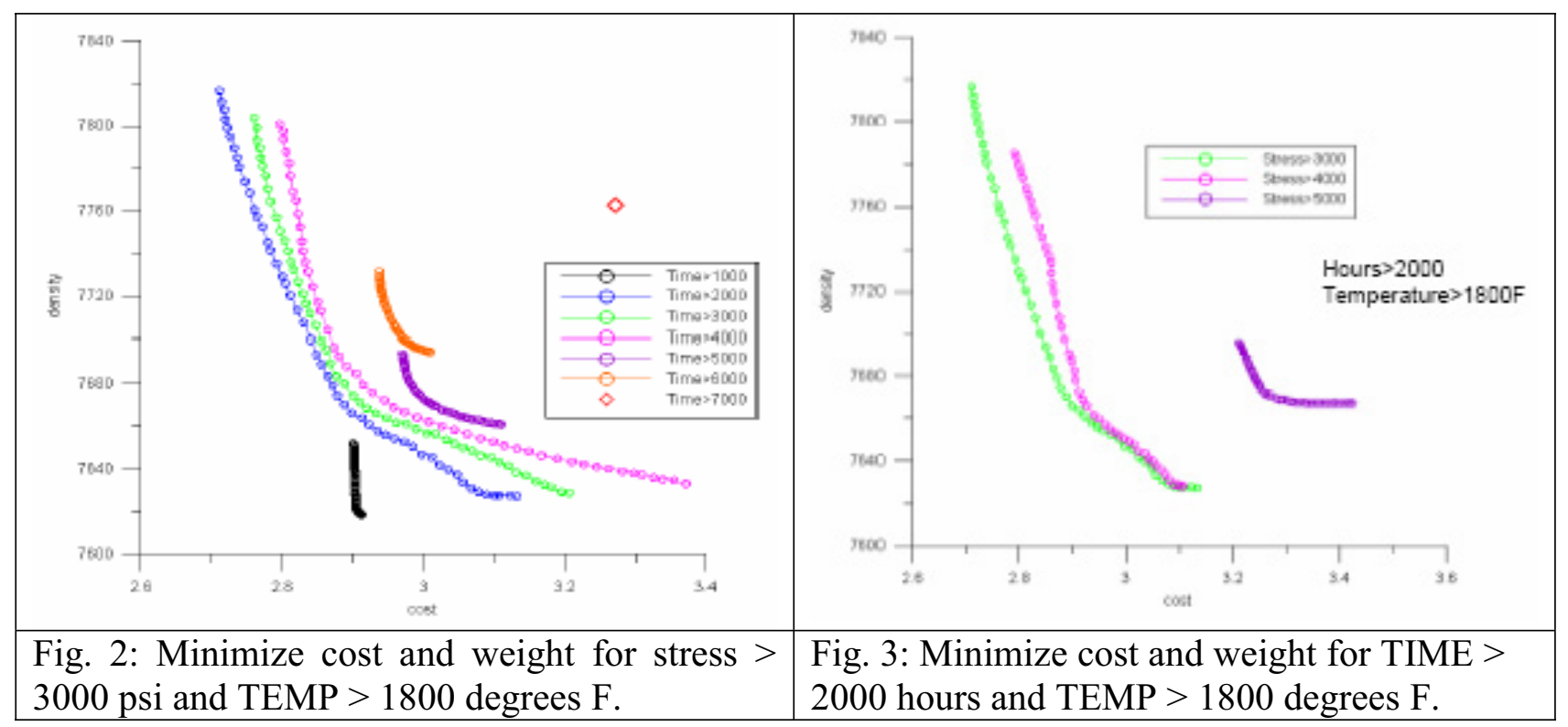


We are hereby publishing experimental data and chemical compositions of all 120 original alloys (Table II) and the 20 optimized alloys (Table III) created in the 4th optimization cycle.
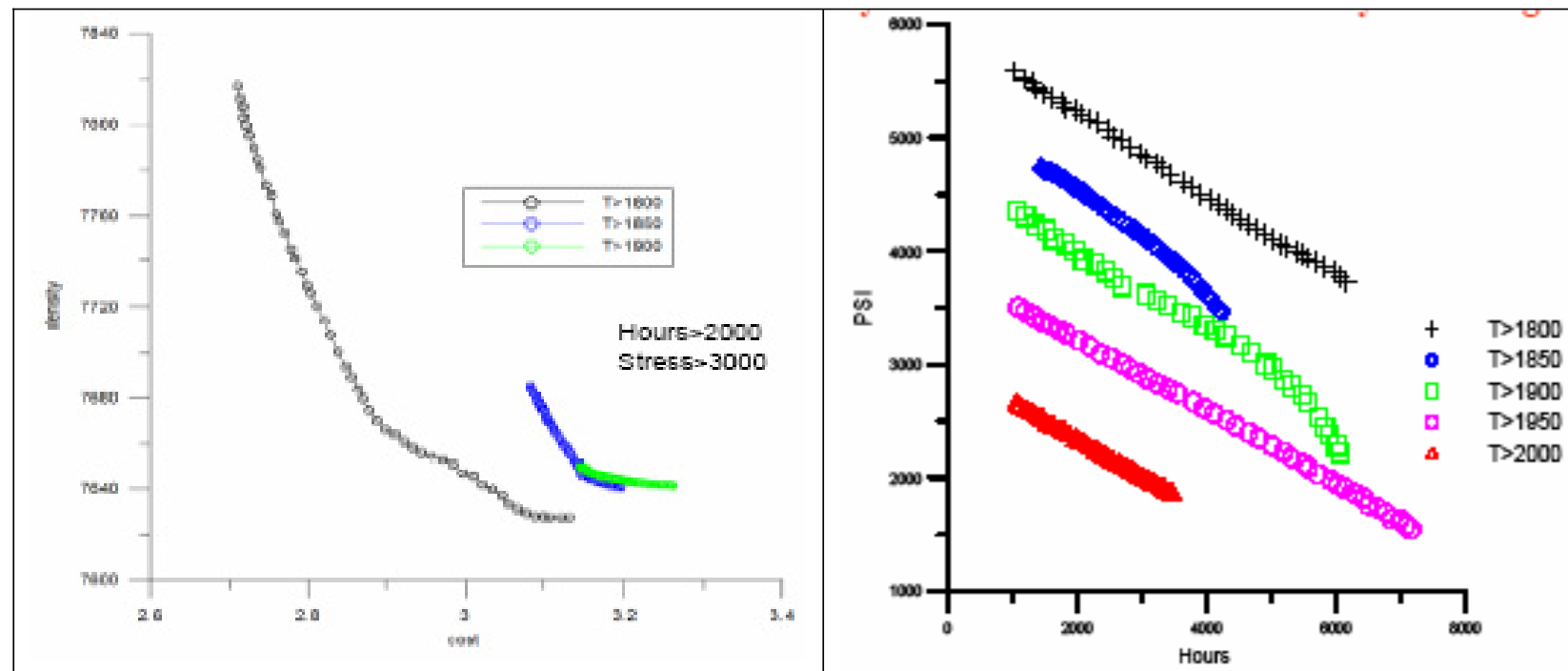

Fig. 5: Minimize cost and weight for stress $>$ 3000 psi and TIME $>2000$ hours.

Fig. 6: Maximize strength and TIME for cost $<$ 3000 and density $<7800 \mathrm{~kg} / \mathrm{m}^{3}$

Table II. Maximum stress at room temperature and time-to-rupture at 975 degrees Celsius and stress of $23 \mathrm{~kg} / \mathrm{mm}^{2}$ for the original set having 120 experimentally tested Ni-base steel alloys.

\begin{tabular}{|c|c|c|c|c|c|c|c|c|c|}
\hline $\begin{array}{c}\text { Alloy } \\
\mathbf{n o} .\end{array}$ & $\begin{array}{c}\text { Sigma, } \\
\mathbf{k g} / \mathbf{m m}^{2}\end{array}$ & $\begin{array}{c}\text { Time, } \\
\text { hours }\end{array}$ & $\mathbf{C}, \mathbf{\%}$ & $\mathbf{C r}, \mathbf{\%}$ & $\mathbf{C o}, \mathbf{\%}$ & $\mathbf{W}, \mathbf{\%}$ & $\mathbf{M o}, \mathbf{\%}$ & $\mathbf{A l , ~ \%}$ & $\mathbf{T i}, \mathbf{\%}$ \\
\hline 1 & 103.7 & 45.02 & 0.190 & 8.93 & 9.90 & 10.23 & 1.27 & 5.67 & 2.45 \\
\hline 2 & 103.7 & 25.17 & 0.175 & 8.75 & 9.93 & 9.79 & 1.42 & 5.40 & 2.79 \\
\hline 3 & 103.5 & 44.52 & 0.179 & 9.24 & 9.83 & 10.11 & 1.36 & 5.58 & 2.72 \\
\hline 4 & 103.1 & 42.28 & 0.172 & 9.17 & 9.71 & 10.51 & 1.69 & 5.24 & 2.72 \\
\hline 5 & 102.4 & 40.10 & 0.194 & 8.63 & 9.67 & 10.37 & 1.55 & 5.29 & 2.74 \\
\hline 6 & 102.4 & 44.35 & 0.190 & 8.57 & 9.42 & 10.44 & 1.61 & 5.44 & 2.81 \\
\hline 7 & 101.9 & 44.77 & 0.192 & 8.91 & 9.93 & 10.19 & 1.47 & 5.33 & 2.68 \\
\hline 8 & 101.7 & 45.12 & 0.193 & 8.69 & 10.23 & 10.20 & 1.68 & 5.17 & 2.70 \\
\hline 9 & 101.6 & 57.40 & 0.176 & 8.66 & 9.53 & 10.48 & 1.53 & 5.52 & 2.68 \\
\hline 10 & 101.5 & 56.67 & 0.170 & 8.61 & 9.85 & 9.71 & 1.67 & 5.23 & 2.67 \\
\hline 11 & 101.4 & 48.60 & 0.173 & 9.23 & 9.87 & 10.35 & 1.32 & 5.83 & 2.75 \\
\hline 12 & 101.1 & 58.03 & 0.187 & 8.69 & 9.51 & 10.57 & 1.60 & 5.29 & 2.86 \\
\hline 13 & 101.0 & 58.15 & 0.183 & 8.90 & 10.07 & 10.11 & 1.45 & 5.41 & 2.59 \\
\hline 14 & 100.9 & 54.12 & 0.192 & 9.08 & 9.84 & 9.86 & 1.40 & 5.80 & 2.73 \\
\hline 15 & 100.8 & 56.02 & 0.157 & 9.01 & 9.77 & 10.38 & 1.25 & 5.45 & 2.66 \\
\hline 16 & 100.7 & 66.32 & 0.168 & 8.86 & 10.05 & 10.05 & 1.51 & 5.52 & 2.67 \\
\hline 17 & 100.6 & 66.55 & 0.186 & 9.23 & 9.74 & 10.02 & 1.46 & 5.68 & 2.72 \\
\hline 18 & 100.5 & 67.45 & 0.162 & 8.94 & 9.79 & 10.07 & 1.40 & 5.55 & 2.79 \\
\hline
\end{tabular}




\begin{tabular}{|c|c|c|c|c|c|c|c|c|c|}
\hline 19 & 100.5 & 67.45 & 0.173 & 8.68 & 9.92 & 10.14 & 1.64 & 5.41 & 2.74 \\
\hline 20 & 100.5 & 68.18 & 0.165 & 9.00 & 9.69 & 10.58 & 1.29 & 5.56 & 2.72 \\
\hline 21 & 100.2 & 71.07 & 0.177 & 9.07 & 9.64 & 9.78 & 1.47 & 5.75 & 2.72 \\
\hline 22 & 100.2 & 71.55 & 0.185 & 8.72 & 9.44 & 10.23 & 1.67 & 5.63 & 2.75 \\
\hline 23 & 100.2 & 64.92 & 0.163 & 8.78 & 9.56 & 10.36 & 1.70 & 5.49 & 2.81 \\
\hline 24 & 100.0 & 69.47 & 0.179 & 9.02 & 9.70 & 10.47 & 1.47 & 5.91 & 2.76 \\
\hline 25 & 99.7 & 76.11 & 0.197 & 8.80 & 9.61 & 10.06 & 1.22 & 5.47 & 2.55 \\
\hline 26 & 99.7 & 67.84 & 0.192 & 8.88 & 9.85 & 9.83 & 1.43 & 5.63 & 2.90 \\
\hline 27 & 99.6 & 76.80 & 0.179 & 9.10 & 9.80 & 10.72 & 1.40 & 5.26 & 2.71 \\
\hline 28 & 99.5 & 72.15 & 0.179 & 8.75 & 9.97 & 9.96 & 1.62 & 5.54 & 2.54 \\
\hline 29 & 99.4 & 64.74 & 0.185 & 9.07 & 10.10 & 9.94 & 1.41 & 5.65 & 2.83 \\
\hline 30 & 99.3 & 60.30 & 0.163 & 8.92 & 9.99 & 10.03 & 1.50 & 5.48 & 2.78 \\
\hline 31 & 99.3 & 33.40 & 0.171 & 9.22 & 9.97 & 10.78 & 1.64 & 5.80 & 2.77 \\
\hline 32 & 99.1 & 80.13 & 0.186 & 8.94 & 9.90 & 10.57 & 1.33 & 5.42 & 2.57 \\
\hline 33 & 99.0 & 76.86 & 0.181 & 8.51 & 9.69 & 10.12 & 1.50 & 5.46 & 2.64 \\
\hline 34 & 99.0 & 81.25 & 0.173 & 8.81 & 9.98 & 10.11 & 1.62 & 5.26 & 2.72 \\
\hline 35 & 99.0 & 39.62 & 0.181 & 9.46 & 9.76 & 10.57 & 1.40 & 5.47 & 2.74 \\
\hline 36 & 99.0 & 81.05 & 0.167 & 8.69 & 9.50 & 10.24 & 1.60 & 5.54 & 2.72 \\
\hline 37 & 98.9 & 79.14 & 0.178 & 8.99 & 9.66 & 10.48 & 1.64 & 5.37 & 2.71 \\
\hline 38 & 98.8 & 80.70 & 0.194 & 8.93 & 9.63 & 9.78 & 1.70 & 5.22 & 2.58 \\
\hline 39 & 98.7 & 74.42 & 0.187 & 8.88 & 9.65 & 10.37 & 1.59 & 5.49 & 2.76 \\
\hline 40 & 98.6 & 64.99 & 0.182 & 8.94 & 10.07 & 9.69 & 1.47 & 5.69 & 2.76 \\
\hline 41 & 98.6 & 69.03 & 0.188 & 8.93 & 9.53 & 10.43 & 1.66 & 5.52 & 2.77 \\
\hline 42 & 98.5 & 72.63 & 0.186 & 8.62 & 9.75 & 10.59 & 1.65 & 5.17 & 2.69 \\
\hline 43 & 98.5 & 77.92 & 0.195 & 8.73 & 9.65 & 10.49 & 1.59 & 5.24 & 2.61 \\
\hline 44 & 98.4 & 85.20 & 0.177 & 8.82 & 9.66 & 10.34 & 1.27 & 5.59 & 2.73 \\
\hline 45 & 98.4 & 80.47 & 0.190 & 8.95 & 9.82 & 10.71 & 1.60 & 5.51 & 2.69 \\
\hline 46 & 98.4 & 77.82 & 0.195 & 8.70 & 9.42 & 10.03 & 1.59 & 5.20 & 2.79 \\
\hline 47 & 98.3 & 41.90 & 0.162 & 8.43 & 9.83 & 10.71 & 1.51 & 5.26 & 2.56 \\
\hline 48 & 98.3 & 65.50 & 0.164 & 8.91 & 9.66 & 10.69 & 1.54 & 5.48 & 2.55 \\
\hline 49 & 98.1 & 73.34 & 0.199 & 9.20 & 10.21 & 9.71 & 1.35 & 5.60 & 2.75 \\
\hline 50 & 98.1 & 65.60 & 0.160 & 8.79 & 9.72 & 10.45 & 1.48 & 5.57 & 2.66 \\
\hline 51 & 98.0 & 84.30 & 0.171 & 8.70 & 10.21 & 10.08 & 1.49 & 5.41 & 2.66 \\
\hline 52 & 98.0 & 78.51 & 0.169 & 9.09 & 9.75 & 10.04 & 1.39 & 5.52 & 2.77 \\
\hline
\end{tabular}




\begin{tabular}{|c|c|c|c|c|c|c|c|c|c|}
\hline 53 & 97.9 & 88.80 & 0.183 & 8.76 & 9.84 & 10.15 & 1.42 & 5.36 & 2.59 \\
\hline 54 & 97.9 & 89.12 & 0.180 & 8.66 & 10.06 & 10.03 & 1.60 & 5.43 & 2.73 \\
\hline 55 & 97.9 & 59.84 & 0.188 & 9.09 & 9.74 & 10.55 & 1.76 & 5.38 & 2.78 \\
\hline 56 & 97.9 & 80.98 & 0.171 & 8.70 & 9.46 & 10.12 & 1.55 & 5.85 & 2.64 \\
\hline 57 & 97.9 & 74.75 & 0.179 & 9.11 & 9.73 & 10.22 & 1.66 & 5.28 & 2.77 \\
\hline 58 & 97.8 & 73.87 & 0.190 & 8.74 & 9.74 & 10.20 & 1.50 & 5.14 & 2.60 \\
\hline 59 & 97.8 & 88.07 & 0.165 & 8.92 & 9.69 & 10.69 & 1.34 & 5.39 & 2.50 \\
\hline 60 & 97.8 & 74.75 & 0.189 & 8.85 & 9.88 & 10.53 & 1.48 & 5.73 & 2.63 \\
\hline 61 & 97.8 & 79.66 & 0.188 & 8.72 & 9.60 & 10.39 & 1.53 & 5.20 & 2.72 \\
\hline 62 & 97.8 & 76.83 & 0.196 & 8.68 & 9.48 & 10.52 & 1.65 & 5.08 & 2.64 \\
\hline 63 & 97.7 & 72.56 & 0.194 & 8.87 & 9.52 & 10.58 & 1.75 & 5.61 & 2.65 \\
\hline 64 & 97.7 & 49.20 & 0.170 & 9.21 & 9.75 & 10.83 & 1.55 & 5.81 & 2.63 \\
\hline 65 & 97.6 & 83.29 & 0.167 & 9.00 & 9.83 & 10.37 & 1.48 & 5.40 & 2.69 \\
\hline 66 & 97.3 & 76.14 & 0.185 & 8.74 & 9.54 & 10.38 & 1.71 & 5.78 & 2.68 \\
\hline 67 & 97.2 & 72.47 & 0.187 & 8.73 & 10.15 & 10.26 & 1.56 & 5.58 & 2.81 \\
\hline 68 & 97.1 & 72.96 & 0.191 & 9.03 & 9.64 & 10.53 & 1.60 & 5.22 & 2.80 \\
\hline 69 & 97.1 & 75.84 & 0.194 & 8.81 & 9.72 & 10.44 & 1.63 & 5.44 & 2.78 \\
\hline 70 & 97.0 & 59.42 & 0.176 & 8.82 & 10.15 & 10.03 & 1.34 & 5.24 & 2.66 \\
\hline 71 & 97.0 & 35.87 & 0.171 & 9.31 & 10.13 & 10.93 & 1.39 & 5.49 & 2.55 \\
\hline 72 & 97.0 & 85.47 & 0.170 & 8.60 & 9.48 & 9.84 & 1.46 & 5.39 & 2.41 \\
\hline 73 & 96.9 & 81.25 & 0.180 & 8.72 & 9.74 & 10.58 & 1.67 & 5.36 & 2.74 \\
\hline 74 & 96.8 & 40.80 & 0.197 & 9.10 & 9.98 & 10.72 & 1.68 & 5.09 & 2.70 \\
\hline 75 & 96.7 & 84.50 & 0.167 & 9.03 & 10.05 & 10.37 & 1.33 & 5.23 & 2.57 \\
\hline 76 & 96.6 & 80.28 & 0.166 & 8.97 & 9.76 & 10.02 & 1.33 & 5.70 & 2.80 \\
\hline 77 & 96.6 & 62.33 & 0.180 & 9.15 & 9.50 & 10.44 & 1.71 & 5.45 & 2.84 \\
\hline 78 & 96.4 & 86.09 & 0.163 & 8.56 & 9.89 & 10.11 & 1.33 & 5.39 & 2.65 \\
\hline 79 & 96.4 & 90.75 & 0.183 & 8.78 & 9.59 & 10.63 & 1.60 & 5.45 & 2.78 \\
\hline 80 & 96.2 & 91.55 & 0.183 & 9.13 & 9.68 & 10.33 & 1.44 & 5.42 & 2.60 \\
\hline 81 & 96.2 & 59.84 & 0.182 & 9.11 & 9.91 & 10.06 & 1.44 & 5.76 & 2.76 \\
\hline 82 & 96.2 & 70.78 & 0.169 & 9.22 & 9.56 & 10.42 & 1.35 & 5.38 & 2.73 \\
\hline 83 & 96.2 & 70.71 & 0.194 & 9.08 & 9.70 & 10.27 & 1.61 & 5.32 & 2.55 \\
\hline 84 & 96.2 & 70.99 & 0.177 & 9.05 & 9.76 & 10.52 & 1.61 & 5.40 & 2.73 \\
\hline 85 & 96.2 & 65.20 & 0.181 & 8.90 & 9.81 & 10.72 & 1.68 & 5.66 & 2.58 \\
\hline 86 & 96.0 & 39.22 & 0.212 & 9.23 & 9.91 & 10.68 & 1.64 & 5.22 & 2.65 \\
\hline
\end{tabular}




\begin{tabular}{|c|c|c|c|c|c|c|c|c|c|}
\hline 87 & 95.9 & 64.91 & 0.186 & 8.66 & 9.43 & 10.37 & 1.54 & 5.26 & 2.81 \\
\hline 88 & 95.8 & 82.13 & 0.174 & 8.65 & 9.76 & 9.73 & 1.40 & 5.32 & 2.52 \\
\hline 89 & 95.8 & 89.53 & 0.163 & 8.79 & 9.79 & 10.40 & 1.43 & 5.34 & 2.56 \\
\hline 90 & 95.8 & 75.10 & 0.197 & 9.04 & 9.83 & 10.62 & 1.57 & 5.39 & 2.77 \\
\hline 91 & 95.7 & 81.71 & 0.163 & 8.64 & 9.83 & 10.55 & 1.45 & 5.11 & 2.56 \\
\hline 92 & 95.7 & 76.69 & 0.179 & 9.24 & 9.78 & 9.90 & 1.38 & 5.39 & 2.75 \\
\hline 93 & 95.6 & 88.91 & 0.189 & 8.75 & 9.79 & 10.14 & 1.30 & 5.34 & 2.47 \\
\hline 94 & 95.6 & 85.94 & 0.185 & 8.88 & 9.93 & 10.56 & 1.41 & 5.30 & 2.52 \\
\hline 95 & 95.6 & 77.96 & 0.175 & 8.73 & 9.58 & 10.51 & 1.57 & 5.67 & 2.73 \\
\hline 96 & 95.6 & 75.40 & 0.192 & 8.79 & 9.51 & 10.37 & 1.55 & 5.40 & 2.62 \\
\hline 97 & 95.5 & 72.05 & 0.182 & 9.07 & 9.48 & 10.39 & 1.78 & 5.33 & 2.67 \\
\hline 98 & 95.5 & 30.30 & 0.179 & 8.68 & 9.63 & 10.42 & 1.60 & 5.67 & 2.85 \\
\hline 99 & 95.4 & 43.19 & 0.175 & 8.82 & 9.56 & 10.45 & 1.65 & 5.60 & 2.87 \\
\hline 100 & 95.4 & 34.50 & 0.152 & 9.00 & 10.06 & 10.78 & 1.64 & 5.73 & 2.63 \\
\hline 101 & 95.3 & 83.56 & 0.182 & 9.20 & 9.75 & 10.06 & 1.45 & 5.04 & 2.64 \\
\hline 102 & 95.3 & 74.43 & 0.188 & 8.81 & 9.41 & 10.47 & 1.61 & 5.48 & 2.60 \\
\hline 103 & 95.3 & 61.25 & 0.168 & 8.85 & 9.26 & 10.15 & 1.55 & 5.45 & 2.72 \\
\hline 104 & 95.2 & 81.88 & 0.177 & 9.15 & 9.70 & 10.42 & 1.26 & 5.47 & 2.56 \\
\hline 105 & 95.2 & 70.91 & 0.189 & 8.80 & 9.53 & 10.40 & 1.56 & 5.93 & 2.72 \\
\hline 106 & 95.1 & 77.05 & 0.182 & 8.70 & 9.77 & 10.18 & 1.38 & 5.50 & 2.72 \\
\hline 107 & 95.0 & 71.07 & 0.183 & 8.98 & 9.73 & 10.49 & 1.64 & 5.48 & 2.63 \\
\hline 108 & 94.9 & 85.00 & 0.178 & 8.72 & 9.84 & 10.27 & 1.50 & 5.47 & 2.67 \\
\hline 109 & 94.6 & 54.65 & 0.183 & 9.00 & 10.24 & 10.55 & 1.53 & 5.44 & 2.65 \\
\hline 110 & 94.5 & 67.43 & 0.193 & 9.13 & 9.75 & 10.65 & 1.61 & 5.21 & 2.72 \\
\hline 111 & 94.5 & 63.30 & 0.169 & 9.22 & 9.69 & 10.29 & 1.40 & 5.52 & 2.77 \\
\hline 112 & 94.2 & 84.46 & 0.173 & 8.88 & 9.98 & 10.83 & 1.41 & 5.31 & 2.59 \\
\hline 113 & 94.1 & 70.17 & 0.184 & 9.06 & 9.44 & 10.23 & 1.66 & 5.92 & 2.70 \\
\hline 114 & 94.0 & 87.63 & 0.182 & 8.97 & 9.61 & 9.86 & 1.24 & 5.63 & 2.87 \\
\hline 115 & 93.9 & 74.83 & 0.174 & 8.95 & 9.89 & 10.21 & 1.42 & 5.18 & 2.75 \\
\hline 116 & 93.5 & 74.73 & 0.198 & 9.03 & 9.88 & 9.69 & 1.33 & 5.58 & 2.84 \\
\hline 117 & 93.2 & 92.38 & 0.168 & 8.77 & 9.95 & 9.91 & 1.58 & 5.48 & 2.70 \\
\hline 118 & 93.0 & 72.70 & 0.157 & 8.91 & 9.77 & 10.17 & 1.46 & 5.51 & 2.80 \\
\hline 119 & 92.7 & 83.60 & 0.178 & 9.08 & 9.55 & 10.52 & 1.65 & 5.60 & 2.69 \\
\hline 120 & 90.7 & 80.10 & 0.173 & 9.00 & 10.07 & 9.88 & 1.38 & 5.77 & 2.83 \\
\hline
\end{tabular}


Table III. Twenty alloys predicted by the optimizer after the 4th optimization iteration and their experimentally evaluated stresses and times until rupture (shaded alloys are true Pareto optimal).

\begin{tabular}{|c|c|c|c|c|c|c|c|c|c|}
\hline $\begin{array}{c}\text { Alloy } \\
\text { no. }\end{array}$ & $\begin{array}{l}\text { Sigma, } \\
\mathrm{kg} / \mathrm{mm}^{2}\end{array}$ & $\begin{array}{l}\text { Time, } \\
\text { hours }\end{array}$ & $\mathrm{C}, \%$ & $\mathrm{Cr}, \%$ & Co, $\%$ & $\mathrm{~W}, \%$ & Мo, \% & $\mathrm{Al}, \%$ & $\mathrm{Ti}, \%$ \\
\hline 181 & 109.7 & 51.7 & 0.177 & 8.94 & 9.53 & 10.51 & 1.58 & 5.38 & 2.70 \\
\hline 182 & 105.8 & 71.0 & 0.189 & 8.88 & 9.58 & 10.64 & 1.63 & 5.47 & 2.78 \\
\hline 183 & 103.3 & 29.4 & 0.194 & 9.24 & 9.95 & 10.68 & 1.72 & 5.31 & 2.66 \\
\hline 184 & 102.8 & 73.9 & 0.190 & 8.98 & 9.70 & 10.55 & 1.76 & 5.52 & 2.70 \\
\hline 185 & 101.5 & 35.0 & 0.172 & 9.03 & 10.04 & 10.65 & 1.73 & 5.50 & 2.82 \\
\hline 186 & 101.3 & 92.1 & 0.178 & 9.04 & 9.93 & 10.60 & 1.52 & 5.33 & 2.55 \\
\hline 187 & 101.3 & 27.7 & 0.194 & 9.12 & 9.74 & 10.64 & 1.69 & 5.32 & 2.66 \\
\hline 188 & 101.1 & 103.9 & 0.179 & 9.13 & 10.10 & 10.09 & 1.67 & 5.71 & 2.66 \\
\hline 189 & 101.0 & 111.3 & 0.170 & 8.87 & 9.66 & 10.40 & 1.58 & 5.63 & 2.73 \\
\hline 190 & 101.0 & 46.1 & 0.159 & 9.01 & 9.54 & 10.64 & 1.67 & 4.89 & 2.60 \\
\hline 191 & 100.7 & 32.7 & 0.178 & 9.40 & 10.17 & 10.40 & 1.74 & 5.76 & 2.78 \\
\hline 192 & 100.6 & 55.7 & 0.164 & 8.79 & 9.79 & 10.67 & 1.57 & 5.53 & 2.59 \\
\hline 193 & 100.5 & 126.3 & 0.178 & 8.91 & 9.70 & 10.28 & 1.55 & 5.45 & 2.67 \\
\hline 194 & 100.4 & 52.5 & 0.177 & 9.03 & 9.85 & 10.55 & 1.72 & 5.57 & 2.62 \\
\hline 195 & 100.1 & 63.2 & 0.170 & 9.04 & 9.58 & 10.30 & 1.58 & 5.42 & 2.56 \\
\hline 196 & 99.8 & 61.5 & 0.166 & 8.84 & 9.87 & 10.25 & 1.69 & 5.54 & 2.72 \\
\hline 197 & 99.6 & 87.2 & 0.168 & 8.78 & 9.97 & 10.60 & 1.52 & 5.36 & 2.67 \\
\hline 198 & 99.6 & 68.4 & 0.192 & 8.78 & 9.58 & 10.55 & 1.61 & 5.64 & 2.66 \\
\hline 199 & 99.2 & 82.8 & 0.157 & 8.90 & 9.70 & 10.58 & 1.50 & 5.45 & 2.68 \\
\hline 200 & 94.5 & 90.0 & 0.173 & 9.12 & 9.60 & 9.94 & 1.25 & 5.71 & 2.83 \\
\hline
\end{tabular}

\section{Summary}

A new method was demonstrated that offers a realistic possibility to significantly reduce the cost and time to predict chemical concentrations and multiple properties of a number of steel alloys so that the new alloys will have superior properties. The new alloy design concept uses a combination of a multi-objective stochastic optimization algorithm and experimental data for thermo-mechanical properties while requiring a minimum number of experimental evaluations of the candidate alloys, in order to verify the computational results. This method has the potential of identifying new alloy compositions that cannot be identified without carrying out an unacceptably large number of experiments if using any other approach. Conceptually, this approach is applicable to design optimization of arbitrary alloys and could be extended to include additional objectives such as thermal treatments and properly quantified grain morphology.

\section{Acknowledgements}

The authors are grateful for the financial support provided for this work by the US Army Research Office under the grant DAAD 19-02-1-0363 monitored by Dr. William Mullins. 


\section{References}

1. H.K.D.H. Bhadeshia, "Neural networks in materials science", ISIJ International, 39 (1999), 966-979.

2. N. Chakraborti, "Genetic Algorithms in Materials Design and Processing", International Materials Reviews, 49 (3-4) (2004) 246-260.

3. H.K.D.H. Bhadeshia and T. Sourmail, "Design of Creep-Resistant Steels: Success \& Failure of Models", Japan Soc. for the Promotion of Science, Committee on Heat-Resisting Materials and Alloys, 44 (2003) 299-314.

4. Kalianmoy Deb, Multi-Objective Optimization Using Evolutionary Algorithms (John Wiley \& Sons, 2002).

5. I.N. Egorov, G.V. Kretinin and I.A. Leshchenko, "Multicriteria Optimization of Time Control Laws of Short Take-Off and Vertical Landing Aircraft Power Plant," ASME paper 97-GT-263 (1997).

6. I.N. Egorov, "Indirect Optimization Method on the Basis of Self-Organization," Proceedings of Optimization Techniques and Applications (ICOTA'98), Curtin University of Technology, Perth, Australia, 2 (1998), 683-691.

7. G.S. Dulikravich, I.N. Egorov, V.K. Sikka and G. Muralidharan, "Semi-Stochastic Optimization of Chemical Composition of High-Temperature Austenitic Steels for Desired Mechanical Properties" (Paper presented at the 2003 TMS Annual Meeting, Yazawa International Symposium: Processing and Technologies) TMS Publication, (eds: Kongoli, F., Itakagi, K., Yamaguchi, C. and Sohn, H.-Y.), Vol. 1, pp. 801-814, San Diego, CA, March 2-6 (2003).

8. I.N. Yegorov-Egorov and G.S. Dulikravich, "Inverse Design of Alloys for Specified Stress, Temperature and Time-to-Rupture by Using Stochastic Optimization", (International Symposium on Inverse Problems, Design and Optimization - IPDO; eds: Colaco, M. J., Orlande, H. R. B. and Dulikravich, G. S.), Rio de Janeiro, Brazil, March 17-19 (2004).

9. I.N. Yegorov-Egorov and G.S. Dulikravich, "Optimization of Alloy Chemistry for Maximum Stress and Time-to-Rupture at High Temperature" (Paper AIAA-2004-4348 presented at $10^{\text {th }}$ AIAA/ISSMO Multidisciplinary Analysis and Optimization Conference, Albany, NY, Aug. 30 Sept. 1 2004).

10. I. N. Egorov-Yegorov and G.S. Dulikravich, "Chemical Composition Design of Superalloys for Maximum Stress, Temperature and Time-to-Rupture Using Self-Adapting Response Surface Optimization", Materials and Manufacturing Processes, 20 (3) (2005), 569-590.

11. G.S. Dulikravich and I.N. Egorov-Yegorov, "Robust Optimization of Concentrations of Alloying Elements in Steel for Maximum Temperature, Strength, Time-To-Rupture and Minimum Cost and Weight" (Paper presented at ECCOMAS - Computational Methods for Coupled Problems in Science and Engineering; eds: Papadrakakis, Onate, E. and Schrefler, B., Fira, Santorini Island, Greece, May 25-28, 2005).

12. I. M. Sobol, "Uniformly Distributed Sequences with an Additional Uniform Property," USSR Computational Mathematics and Mathematical Physics, 16 (1976), 236-242. 